\title{
UAHSP :
}

\section{The Internet Journal of Allied Health Sciences and Practice}

A Peer Reviewed Publication of the College of Health Care Sciences at Nova Southeastern University

Dedicated to allied health professional practice and education

http://ijahsp.nova.edu Vol. 12 No. 3 ISSN 1540-580X

\section{A Qualitative Evaluation of Blood Pressure Screening Procedures in the Illinois WISEWOMEN Program}

\author{
James Broadbear, PhD ${ }^{1}$ \\ Barbara Broadbear, $\mathrm{PhD}, \mathrm{MPH}^{2}$ \\ Adrian Lyde, $\mathrm{PhD}^{3}$ \\ Sharon Mills, MS ${ }^{4}$
}

1. Professor of Health Education, Department of Health Sciences, Illinois State University, Normal, IL

2. Adjunct Professor, Health and Human Services Division, Heartland Community College, Normal, IL

3. Assistant Professor of Health Education, Department of Health Sciences, Illinois State University, Normal, IL

4. Director, Applied Social Research Unit, Illinois State University, Normal, IL

United States

CITATION: Broadbear J, Broadbear B, Lyde A, Mills S. A Qualitative Evaluation of Blood Pressure Screening Procedures in the Illinois WISEWOMEN Program. The Internet Journal of Allied Health Sciences and Practice. July 2014. Volume 12 Number 3.

\section{ABSTRACT}

Hypertension is a major modifiable risk factor for cardiovascular disease and various other conditions. Approximately 70 million Americans are hypertensive. Among women age 18 and over, 31.7\% have high blood pressure (BP) and only about $60 \%$ of those women have it under control. The purpose of this evaluation was to understand blood pressure screening procedures among Illinois WISEWOMAN Program (IWP) providers. Procedures at primary care providers (PCP) compared to obstetrician/gynecologists $(\mathrm{OB} / \mathrm{GYN})$ were of interest. A qualitative approach was used featuring interviews with allied health staff members who conduct blood pressure screenings. The focus of the interviews included the use and understanding of The Seventh Report of the Joint National Committee on Prevention, Detection, Evaluation, and Treatment of High Blood Pressure (JNC 7) guidelines, BP screening procedures, and perceptions of the IWP. Twenty allied health professionals who conduct blood pressure screenings with IWP participants were interviewed. Transcripts of these interviews were made and a detailed analysis was conducted.

Findings include:

- Providers reported vague or no understanding of the JNC 7 guidelines.

- Providers were not able to accurately describe BP measurement classifications.

- Screening procedures were not aligned with JNC 7 guidelines.

- Providers reported inconsistent procedures for taking a second BP as indicated in the JNC 7 guidelines.

- Re-training in proper BP screening procedures was uncommon.

- When patients presented with elevated blood pressures a trend was clear: OB/GYN refer - PCP treat.

- $\mathrm{OB} / \mathrm{GYN}$ and PCP reported support for the program and saw value for their patients.

Implications of the findings for women's health and recommendations for allied health practice are provided. 


\section{INTRODUCTION}

Hypertension is a well-established major modifiable risk factor for cardiovascular disease. Approximately 70 million Americans are hypertensive including over 3 million adults in Illinois. ${ }^{1,2}$ Many others have never been properly screened. Nearly 1 in 3 (31.7\%) American women age 18 and over suffer from hypertension, and only $60 \%$ of them have it controlled. ${ }^{1}$ Compared to men, women are less likely to have achieved appropriate control.3,4

Disparities are evident in access to BP screenings in the United States. Although $90.6 \%$ of adults had their blood pressure checked within the previous 2 years, only $76.6 \%$ of underinsured or uninsured women had been screened. ${ }^{5,6}$ Hypertension disproportionately affects Hispanic and African American women and those without health insurance. ${ }^{7-9}$

Lifetime accumulating health effects of suboptimal blood pressure control are significant. Undiagnosed or poorly controlled hypertension substantially increases risk for coronary heart disease, congestive heart failure, cerebrovascular disease, dementia, retinopathy, kidney failure, and other conditions. In women, uncontrolled systolic blood pressure (SBP) rises consistently after age 30 from slightly over $110 \mathrm{mmHg}$ to nearly $150 \mathrm{mmHg}$ by age $80 .{ }^{10}$ Sub-optimal control of high SBP results in elevated risk that could be avoided for millions of women.

Widespread community-based blood pressure screening and monitoring can be useful in identifying women at risk for hypertension and can increase diagnoses and treatment in underserved populations. ${ }^{10}$ For example, Ehrenthal et al reported that obstetrician-gynecologists $(\mathrm{OB} / \mathrm{GYN})$ recognized their primary or exclusive role as health care providers for women and that they have an important role in cardiovascular disease prevention. ${ }^{11}$ Improved screening procedures were recommended as a useful tool in enhancing that role. However, screenings alone are not the solution to this growing problem. Schmittdiel et al reported that women under the age of 50 whose hypertension was detected in OB/GYN clinics were less likely to receive treatment compared to women whose hypertension was detected in medical clinics. ${ }^{12}$ An expanding and ongoing need for quality screening and treatment efforts for women - especially those underserved by health care professionals - is evident.

One such effort is the Illinois WISEWOMAN Program (IWP) that provides community-based cardiovascular health screenings for underserved populations. WISEWOMAN is an acronym for "Well-Integrated Screening and Evaluation for Women Across the Nation." It is a federally funded program, administered by state public health agencies, and conducted by local health agencies in collaboration with health care providers. The CDC funds 21 state-run programs throughout the country with a mission "to provide low-income, underinsured, or uninsured 40- to 64-year-old women with the knowledge, skills, and opportunities to improve their diet, physical activity, and other life habits to prevent, delay, or control cardiovascular and other chronic diseases."13 To become a WISEWOMAN provider, a state public health agency must first be part of the National Breast and Cervical Cancer Early Detection Program. Each of the 21 state-run programs selects local health agencies to operate the program in communities. These local health agencies, in turn, enlist health care providers to conduct screenings and interventions with eligible women.

The IWP aspires to meet the needs of women living in diverse geographic settings, from the metropolitan area of Chicago to rural counties with fewer than 40,000 residents. The Illinois Department of Public Health contracts with nine agencies - seven local health departments, one federally-qualified health center, and one hospital - to conduct the program in 15 counties. ${ }^{14}$ These agencies recruit local OB/GYN and primary care providers (PCP) to implement the program with women who qualify. IWP participants are offered cardiovascular screenings and a four-session educational program designed to increase physical activity, improve dietary behaviors, and enhance goal setting. ${ }^{15}$

The purpose of this evaluation was to understand blood pressure screening procedures among IWP providers in OB/GYN and PCP clinics. Qualitative interviews were conducted with allied health staff members at offices serving IWP participants. One focus was the use and understanding of guidelines in the Seventh Report of the Joint National Committee on Prevention, Detection, Evaluation, and Treatment of High Blood Pressure (JNC 7). JNC 7 guidelines established the following standards for classifying BP:

Normal: SBP $<120$ and DBP $<80$;

Prehypertension: SBP 120 to 139 or DBP 80 to 89 ;

Stage 1 Hypertension: SBP 140 to 159 or DBP 90 to 99;

Stage 2 Hypertension: SBP 160 or above or DBP 100 or above. ${ }^{10}$

The guidelines also describe proper BP screening procedures including averaging two readings to establish a person's blood pressure. Additional points of emphasis in the interviews were how OB/GYN and PCP handled elevated readings, and providers' perceptions of the IWP.

(c) The Internet Journal of Allied Health Sciences and Practice, 2014 


\section{METHODS}

\section{Data Collection}

All procedures received institutional review and approval before the evaluation began. In order to fulfill the focus and purpose of the evaluation, a semi-structured interview format was used. Interviewers were not limited to specific questions but explored participants' experiences with follow-up questions to generate deeper and more precise understandings. ${ }^{16}$ The authors and personnel at the Illinois Department of Public Health collaborated to develop an initial draft of interview topics and questions. The interview guide was further refined through a series of drafts. The final interview guide addressed three major topics including participants':

1. understanding and use of JNC 7 guidelines (awareness of the guidelines, training in BP screening, knowledge of BP classifications);

2. blood pressure screening procedures (taking two blood pressures and averaging the readings, what happens if a patient has an elevated reading); and

3. perceptions of the IWP.

Two interviewers collected the data. Each was well-qualified with significant experience conducting qualitative interviews. All interviews were conducted over the phone. Interviews were digitally recorded and detailed transcripts were produced for analysis.

\section{Participants}

Participants were allied health professionals who perform blood pressure screenings with IWP clients. Interviewers contacted clinics - typically speaking with an office administrator - to explain the project and identify potential participants. When the administrators were receptive to helping interviewers connect with potential participants, follow-up calls were made to schedule phone interviews. The goal was to conduct approximately twenty interviews with a stipulation that this number could be increased depending on an interim appraisal of the results. If several interviews produced a wide breadth of responses, more than 20 respondents may have been needed. A similar number of OB/GYN and PCP were to be interviewed.

\section{Data Analysis}

Two interim appraisals of results were conducted. These appraisals were done after seven and 14 of the 20 interviews were completed. This involved discussing data collection procedures to assure the interviewers were maintaining consistent approaches and developing a preliminary understanding of the results. After the second appraisal, it was determined that the target of approximately 20 interviews was sufficient to generate meaningful results.

Transcripts were made of the interviews and results were analyzed in a three-step process. Initially, all transcripts were read and quotes of interest were identified. A second reading focused on categorizing each of the identified quotes to coincide with the primary topics of the interviews. Finally, themes that emerged from the analysis were identified and organized and quotes illustrating each theme were noted.

\section{RESULTS}

The purpose of this evaluation was to understand blood pressure screening procedures among IWP providers in women's health and primary care settings. The focus of the interviews included participants' understanding of the JNC 7 guidelines, blood pressure screening procedures, and perceptions of the IWP.

Twenty allied health professionals were interviewed. Eight worked in OB/GYN settings and 12 were in PCP settings. All were females. Occupations of the participants included nurses, nurse practitioners, certified nursing assistants, medical assistants, and physician assistants.

Results are presented below and include a brief description of each interview topic followed by illustrative quotations from providers. Quotes are presented in bulleted lists and for most topics are divided between OB/GYN and PCP. 


\section{Understanding of the JNC 7 Guidelines \\ Awareness of the Guidelines}

OB/GYN: Only one provider was aware of the JNC 7 document prior to being interviewed. All others indicated little or no awareness of the document.

- No I am not familiar with them. I don't know what they are. I know BPs and everything but I have never heard of that particular set of guidelines.

PCP: One reported actually reading the document and a few others were aware of the guidelines. While some knowledge emerged during interviews that may have originated in the guidelines, providers in primary care settings generally did not know the source document.

- Yes. We follow them. That is part of our policy.

- Am I familiar with it? No, not really.

- I was not aware of these until I heard that I would be participating in this interview.

\section{Training in Blood Pressure Screening}

Every participant received training on measuring blood pressures while in her professional preparation program. However, only one of the 20 providers reported any form of continuing education related to measuring BP. In many cases, it had been 20 years or more since being trained.

OB/GYN

- I haven't had any specific training on how to check BPs since nursing school.

- Taking me back to 1965 , that's when I learned. No training since.

- I can't think of any (continuing education) specifically pertaining to hypertension or blood pressure.

PCP

- No training (beyond school) that I can remember.

- Did we get any additional training at the job where I am? No.

\section{Knowledge of Blood Pressure Classifications}

Providers were asked to identify the classifications of normal BP, prehypertension, and stage 1 and 2 hypertension. It was assumed that health care professionals who regularly conduct blood pressure screenings would be knowledgeable of the classifications. This was not the case. Knowledge of $\mathrm{BP}$ classifications was vague among all providers. Only one OB/GYN provider and two PCP correctly identified all four classifications. The rest identified normal blood pressure as 120/80 or below rather than the more precise below 120/80. Knowledge of the other three classifications was inconsistent. Participants typically described hypertension as 140/90 or above. Precise knowledge about classifications was lacking among nearly all of the providers. The following quotes represent the lack of accurate knowledge of the guidelines:

- A BP less than 140 over 90 is normal.

- I am aware of recommendations for normal BPs being a little lower than previously with the high being 135 for systolic.

- Hypertension stage 2: I would go like 160 to 170 over 90.

- Hypertension stage 2: ...just anything above 150 over 100.

- ...prehypertension would be anywhere from like about 140 , maybe 90 , right around that range.

\section{Blood Pressure Screening Procedures}

A focus of the evaluation was whether IWP providers followed the guidelines for conducting screenings by properly performing at least two measurements and recording the average. Several participants reported taking a second BP if the first one was elevated. Some providers reported taking two blood pressures - one in each arm or having the patient in different positions such as seated and lying down. No providers indicated taking two blood pressures and averaging the readings - as indicated in the guidelines. 


\section{Second Blood Pressure}

OB/GYN

- We record their BP twice. And we take it on the right and the left.

- Sometimes it's busy in the office. In that case, we only do it if it's high or we think it's not normal.

- Through the IWP we are told to take two BPs, at the beginning of the visit and right before the patient leaves, re-check the BP.

- Only if it's elevated. If you get an elevated BP over 136 over 80 . I usually lay them on their left side for about five minutes and repeat the $\mathrm{BP}$ on both sides.

- If a patient has a high BP reading, I have (them) sit 10 minutes. Sometimes it's impossible when it gets crazy busy to actually have them sit 10 minutes.

- I have them sit up straight and make sure their legs aren't crossed, if it's high, l'll have the patient lie down, take it again. If it's still high, stand up. Those three ways.

PCP

- I think it's a time factor. If we don't have the (IWP) form with us, we may not realize that they need to do two BPs.

- If it's out of range we have them rest a bit then recheck it.

- In terms of diagnosis we don't take the first blood pressure we get, just to be sure we have a second or third visit to double check the reading.

- We take a second pressure when it's (above) 150 over 90 or if they come in here with it like 90 or higher on the diastolic.

- On the WISEWOMAN we have guidelines to take a second blood pressure. There is a form to fill out. It requires you to take a second BP ten minutes after the first BP.

- We do one on each arm.

\section{Elevated Blood Pressure Readings}

There was a clear distinction between OB/GYN and PCP regarding how patients with elevated blood pressure were handled. $\mathrm{OB} / \mathrm{GYN}$ providers generally refer patients elsewhere for follow-up and treatment. There were providers who indicated they offered some level of treatment but that was atypical. Primary care providers treat hypertension. The contrast in approaches is evident in the following quotes.

OB/GYN

- We automatically refer them to their primary. If they don't have a doctor, I refer them to one of our practitioners here at the office.

- If somebody has a really high blood pressure we would refer them to their primary care physician, assuming they have one, which is not always the case with the WISEWOMAN program. In that case, we give them a list of doctors or clinics they could go to for care.

- ...as OB/GYN practitioners, we do not treat high blood pressure so we talk with the patient, maybe give some tips, but in terms of actually making the diagnoses and treating hypertension, that is outside the scope of our practice.

- ...the patient would either be asked to come back in a week or several days, or referred to their primary health provider.

- I work in a gynecology clinic, so we're not primary care providers. We don't treat hypertension. Sometimes the difficult part for us is getting these patients referred to someone that will take them.

- If a patient's BP is extremely high, like stroke time, if that patient does not have a primary and refuses to go to the ER, he may for that one time write them something until they can get into a primary. We don't want them to walk out of here and stroke out when it was something we could have prevented.

PCP

- If it's still high the doctor goes in and talks to them to see if their meds need to be upped or downed.

- He'll have them come back in a week or two and manage it a lot closer and rearrange the medicines until the BP is in normal range.

- If it's really high, we probably start the patient on medication and get a baseline EKG.

- ...it depends, if they really have hypertension we have them take their BP at home then have them come back.

- If elevated we have the patient come in at least two more times over the next two weeks. It requires that you have three different elevated BPs before you can diagnose hypertension. You can't do it in one visit. If elevated on three 
different readings and (the doctor) diagnoses hypertension she discusses lifestyle modification, any risk factors such as diet, smoking, and exercise.

\section{Perspectives of the Illinois WISEWOMAN Program}

The final area of inquiry was about the providers' experiences with IWP. They were queried about their opinions of the program.

\section{OB/GYN}

- I think it's a great program. It provides evaluations for women who don't have access to insurance who need care and explains the importance of regularly getting evaluated by your primary physician.

- You are not going to kill the program are you? We need it.

- I have been doing this for eight years and really enjoy it.

- ...it's very difficult for people who have a specialty rather than primary care or internal medicine to keep up to date with all the other things that go on in the primary care setting. It may be less likely that a specialist is up to date on new guidelines. (BP) is not something that they manage, that they diagnose, something they deal with on a day-to-day basis.

PCP

- ...wish (we saw) more women who had access to WISEWOMAN...there are uninsured women who could benefit from it who don't have access or don't know about it.

- I see how this program really helps women who don't have insurance or money to get these tests done...they really appreciate it.

- I think (IWP) is doing a pretty good job and it's good for our community because there are a lot of people with hypertension in the area that are untreated. When they come in to get that visit and find out, "I have high blood pressure" ...that helps a lot.

\section{DISCUSSION}

In the evaluation of blood pressure screening in the Illinois WISEWOMAN Program, allied health professionals who routinely conduct screenings were interviewed. The evaluation was designed to generate a better understanding of providers' knowledge of the JNC 7 guidelines, how screenings were conducted, and perceptions of IWP.

Few participants demonstrated precise knowledge of BP classifications. Knowledge of normal, prehypertensive, and stage 1 and 2 hypertension was lacking. While the actual diagnosis of hypertension is the responsibility of physicians, allied health professionals involved in blood pressure screenings should know this information so they can provide better service to patients. At a minimum, they could have and use the BP classifications reference card available through the National Heart Lung and Blood Institute web site.

Screening procedures were not closely aligned with JNC 7 guidelines. Accuracy of measurement is highly dependent upon the screening process. Precision can be gained by careful use of the guidelines. This is particularly important in measuring systolic blood pressure which is sensitive to stress, exercise, consumption of caffeine, tobacco or alcohol, and emotional state. Careful adherence to proper screening procedures increases accuracy.

In this study, OB/GYN and PCP did not properly take a second blood pressure and average the results per the JNC 7 guidelines. Providers did report taking a second BP, one in each arm, in different postures, or if the first reading was elevated. However, if the common practice is to take two blood pressures only if the first one is elevated and then presume that a lower reading is accurate, providers are risking false negative results. Averaging the two readings as the guidelines instruct is a more clinically precise approach.

When patients had elevated BP measurements, one trend was evident: PCP treat while OB/GYN refer. This represents a care opportunity lost. For some IWP participants, their OB/GYN is their primary care provider or the only provider they are likely to see. Assuming patients will follow up creates barriers to proper diagnosis and treatment. Screening is the first step in this process and needs to be integrated within the continuum of care. Exploring ways to enhance the role and more fully engage $\mathrm{OB} / \mathrm{GYN}$ in the detection, treatment, and management of hypertension is needed. $., 11,12$

This study was limited in several ways. Twenty allied health professionals who serve IWP participants and were willing to be interviewed over the phone were included. The relatively small number and self-selective nature of the sample were limitations. The qualitative approach provided information about blood pressure screening procedures by these providers but is not

(C) The Internet Journal of Allied Health Sciences and Practice, 2014 
generalizable to all WISEWOMAN providers or other allied health professionals. Not all aspects of proper blood pressure screening procedures were discussed during the interviews. Discussion was primarily limited to providers' understanding of the JNC 7 blood pressure classifications and whether two readings were conducted with the results averaged. Additional research on the professional preparation of allied health professionals and the procedures they use to conduct blood pressure screenings is needed.

Continuing education for all health care providers involved in blood pressure screenings is indicated. Allied health professionals reported training during their original professional preparation, which may have been many years prior - in some cases over 30 years. A lack of continuing education could be a primary reason for inadequate knowledge and procedures reported by participants in this evaluation. Continuing education focused on the JNC 7 guidelines could reinforce important skills and accentuate the need for sound procedures to improve the accuracy of results. Revision of the JNC 7 guidelines is pending. Although updated in 2012, in June 2013 the NHLBI began collaborating with the American Heart Association to produce revised guidelines. ${ }^{17}$

Attention to the process and implementation of the new guidelines is of critical importance to successful blood pressure control. An emphasis on understanding blood pressure classifications and establishing procedures for averaging two properly collected readings would enhance accuracy. Making the detection of high blood pressure a priority can assist in identifying patients in need of lifestyle modification and possible treatment.

\section{REFERENCES}

1. Centers for Disease Control and Prevention. Vital signs: prevalence, treatment, and control of hypertension --- United States, 1999 - 2002 and 2005 - 2008. MMWR [Internet]. 2011 [cited 2013 Oct 29];60(4):103-8. Available from: http://www.cdc.gov/mmwr/pdf/wk/mm6004.pdf.

2. Illinois Department of Public Health. Illinois Behavioral Risk Factor Surveillance System - Hypertension Control. 2012. Available from: http://app.idph.state.il.us/brfss/statedata.asp.

3. Gu Q, Burt VL, Paulose-Ram R, Dillon CF. Gender differences in hypertension treatment, drug utilization patterns, and blood pressure control among U.S. adults with hypertension: data from the National Health and Nutrition Examination Survey 1999-2004. Am J Hypertens. 2008;21(7):789-98. doi: 10.1038/ajh.2008.185. Epub 2008 May 1. [PMID 18451806]

4. Keyhani S, Scobie JV, Herbert PL, McLaughlin MA. Gender disparities in blood pressure control and cardiovascular care in a national sample of ambulatory care visits. Hypertension. 2008;51(4):1149-55. doi: 10.1161/HYPERTENSIONAHA.107.107342. Epub 2008 Feb 7. [PMID 18259013]

5. U.S. Department of Health and Human Services. Healthy People 2020 [Internet]. 2012. Available from: http://www.healthypeople.gov/2020/default.aspx.

6. Robbins CL, Dietz PM, Bombard JM, Gibbs F, Ko JY, Valderrama AL. Blood pressure and cholesterol screening prevalence among U.S. women of reproductive age: opportunities to improve screening. Am J Prev Med. 2011;41(6): 588-95. doi: 10.1016/j.amepre.2011.08.010. [PMID 22099235]

7. Arslan C, Savucu Y, Ceviz D. Evaluation of the body composition, blood lipids and health life-style in employment and unemployment women. Healthmed. 2011;5(4):699-710.

8. Derby CA, FitzGerald G, Lasser NL, Pasternak RC. Application of national screening criteria for blood pressure and cholesterol to perimenopausal women: prevalence of hypertension and hypercholesterolemia in the Study of Women's Health Across the Nation. Prev Cardiol. 2006;9(3):150-9. [PMID 16849878]

9. National Center for Health Statistics. Health Indicators Warehouse [Internet]. 2012; Available from: http://www.healthindicators.gov/Indicators/Highbloodpressurecontrol_882/Profile/Data.

10. National Heart, Lung, and Blood Institute (NHLBI). National Institutes of Health. The Seventh Report of the Joint National Committee on Prevention, Detection, Evaluation, and Treatment of High Blood Pressure. NIH Publication Number 04-5230.

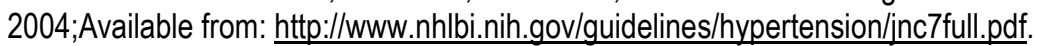

11. Ehrenthal DB, Núñez AE, O'Neill E, Robertson-James C, Addo SF, Stewart A. The role of the obstetrician/gynecologist in the prevention of cardiovascular disease in women. Womens Health Issues. 2011;21(5):338-44. doi:10.1016/j.whi.2011.04.012 [PMID 21703866]

12. Schmittdiel J, Selby JV, Swain B, Daugherty SL, Leong TK, Ho M, Margolis KL, O'Connor P, Magid DJ, Bibbins-Domingo K. Missed opportunities in cardiovascular disease prevention? Low rates of hypertension recognition for women at medicine and obstetrics-gynecology clinics. Hypertension. 2011;57(4):717-22. doi: 10.1161/HYPERTENSIONAHA.110.168195. Epub 2011 Feb 21. [PMID 21339475]

13. Centers for Disease Control and Prevention. WISEWOMAN's Vision and Mission [Internet]. 2011;Available from: http://www.cdc.gov/wisewoman/vision_mission.htm. 
14. Centers for Disease Control and Prevention. WISEWOMAN Program Locations - Illinois [Internet]. 2012;Available from: http://www.cdc.gov/wisewoman/locations/illinois.htm.

15. Khare MM, Carpenter RA, Huber R, Bates NJ, Cursio JF, Balmer PW, Nolen KN, Hudson H, Shippee SJ, Loo RK. Lifestyle Intervention and Cardiovascular Risk Reduction in the Illinois WISEWOMAN Program. J Womens Health (Larchmt). 2012;21(3):294-301. doi: 10.1089/jwh.2011.2926. Epub 2011 Dec 2. [PMID 22136298]

16. Seidman I. Interviewing as qualitative research: a guide for researchers in education and social sciences (4th $\mathrm{ed}$.). 2012;New York: Teachers College Press.

17. Gibbons GH, Shurin SB, Mensah GA, Lauer MS. Refocusing the agenda on cardiovascular guidelines: an announcement from the National Heart, Lung, and Blood Institute [Internet]. 2013;Available from: http://circ.ahajournals.org/content/early/2013/06/18/CIRCULATIONAHA.113.004587. 